\title{
Inhibitory Effects of Nigella sativa Seed Extract on Adrenaline-Induced Dyslipidemia and Left Ventricular Hypertrophy in Rats
}

\author{
Y. Ali ${ }^{1}$, M. S. Islam ${ }^{1}$, A. H. M. K. Alam ${ }^{1}$, M. A. A. Rahman ${ }^{1}$, Al Mamun ${ }^{1}$, M. K. Hossain ${ }^{2}$, \\ A. K. M. M. Hossain ${ }^{1}$, M. S. Parvin ${ }^{1}$, and M. Rashid ${ }^{1 *}$ \\ ${ }^{1}$ Department of Pharmacy, University of Rajshahi, Rajshahi-6205, Bangladesh \\ ${ }^{2}$ Department of Biochemistry and Molecular Biology, University of Rajshahi, Rajshahi-6205, \\ Bangladesh
}

Received 16 October 2012, accepted in revised form 18 March 2013

\begin{abstract}
The aim of this study was to investigate the inhibitory effect of Nigella sativa seed extract (abbreviated as NSSE) on adrenaline-induced dyslipidemia and left ventricular hypertrophy $(\mathrm{LVH})$ in rats. Intraperitoneal injection of adrenaline for two weeks induced dyslipidemia whereas injection of adrenaline for four weeks induced both dyslipidemia and LVH significantly, compared with normal rats. Injection of NSSE with different doses $(0.5,1$ and $1.5 \mathrm{mg} / \mathrm{kg}$ body weight, BW) on adrenaline-induced dyslipidemic rats (AIDRs) for two weeks significantly reduced TC, TG and LDL-C and increased HDL-C compared with AIDRs and the most effective dose was $1.5 \mathrm{mg} / \mathrm{kg} \mathrm{BW}$. Treatment with NSSE $(1.5 \mathrm{mg} / \mathrm{kg}$ BW) on AIDRs for eight weeks, significant anti-dyslipidemic effect was observed. Moreover, NSSE significantly increased free radical scavenging activity and attenuated the LVH and cardiomyocyte size compared with AIDRs. The results suggested that the antidyslipidemic, antioxidative and inhibitory effects of NSSE on LVH were closely resembled to that of atorvastatin, a most effective lipid lowering agent. Therefore, NSSE might be used as a therapeutic alternative or as a combination with drug in the treatment of dyslipidemia and LVH.
\end{abstract}

Keywords: Adrenaline; Dyslipidemia; LVH; NSSE; Atorvastatin.

(c) 2013 JSR Publications. ISSN: 2070-0237 (Print); 2070-0245 (Online). All rights reserved.

doi: http://dx.doi.org/10.3329/jsr.v5i2.12203 J. Sci. Res. 5 (2), 325-334 (2013)

\section{Introduction}

Dyslipidemia, one of the important risk factors for cardiovascular disease (CVD), is a serious public health problem in the world. Several clinical and epidemiological studies suggested that dyslipidemia predisposed atherosclerosis undoubtedly which can lead to hypertension, coronary artery disease (CAD), myocardial infarction (MI) and cerebrovascular and peripheral vascular diseases [1]. It has been reported that dyslipidemia increases mass of the left ventricular myocardium known as left ventricular hypertrophy $(\mathrm{LVH})$ as well as the cardiac myocyte size [2]. LVH and increased cardiac

\footnotetext{
*Corresponding author: mamun69jp@yahoo.com
} 
myocyte size are independent cardiovascular risk factor for myocardial infraction, stroke and sudden cardiac death [3].

Dyslipidemia is associated with the increased production of oxygen free radicals, known as oxidative stress or impaired antioxidant defenses. Oxidative stress leads to apoptosis or myocardial cell injury and dysfunction by alteration of gene expression and modification of cellular responses [4]. Chronic and acute overproduction of reactive oxygen species under pathophysiologic conditions is integral in the development of CVD. It has been reported that increased oxygen free radicals and decreased vitamin E levels have also been demonstrated in patients undergoing coronary artery bypass graft surgery [5]. All of these clinical studies provide support for the concept that increased oxidative stress and decreased antioxidant defenses may play a role in the pathogenesis of CVD. Short term intaperitoneal injection of adrenaline induced dyslipidemia and hypertension in rats [6-8]. However, administration of adrenaline for several weeks to rats can stimulate heart protein synthesis, induce cardiac hypertrophy and alter the sensitivity to ischemia [8$10]$.

Diets play an important role in the management of dyslipidemia. Some studies indicated that increased dietary fiber aids in lipid reduction, possibly by trapping and eliminating cholesterol within the intestinal contents. There are several synthetic drugs that lower abnormally elevated plasma lipoproteins by reducing their production or enhancing their removal. But none of the lipid-lowering drugs is completely satisfactory. Derivatives of fibric acid and nicotinic acid possess some forms of adverse effect and are costly, hence the use of these drugs are limited [11]. Statins such as atorvastatin, simvastatin, etc are another group of drugs used to treat dyslipidemia [12]. However, both fibrates and statins are very costly, possess some degree of adverse effects, hence the search for new, safe and easy to administer drugs remains a top priority. Drugs derived from medicinal plants have been brought into focus for meeting the goals of a wider coverage of health care delivery including dyslipidemias to all countries of the world. Therefore our goal is to search new leads from medicinal plant that could be cheap, easily available and presumably less toxic.

Nigella sativa Linn. commonly known as black seed (local name: kalajira), is a spicy, annual herb, belongs to the family Ranuculaceae [13]. This herb has been known to exhibit potential medicinal properties in folkloric medicine [14]. Ethanolic extract of NS seed was shown to posses cytotoxic and immunopotentiating effect [15] and in the treatment of blood, liver, kidney and heart diseases [16]. The unsaturated fatty acids present in NS may also provide antieicosanoid and antioxidant activities [17]. However, there is no published report about the effect of $N S$ seed on dyslipidemia associated LVH. Therefore, we designed our present study to investigate the inhibitory effects of Nigella sativa seed extract on adrenaline-induced dyslipidemia and LVH in rats.

\section{Materials and Methods}

\subsection{Collection of NS seeds}

Seeds of NS were purchased from a local herbal grocery from Rajshahi city in August, 2010 and were botanically authenticated by a plant taxonomist from the Department of 
Botany, University of Rajshahi, Rajshahi, Bangladesh. A voucher specimen is deposited at the Department of Botany, University of Rajshahi, Bangladesh.

\subsection{Preparation of crude seed extract}

The crude ethanolic extract of NS seed was prepared according to Alam et al., (2002) [18]. In brief, the seeds were cleaned and dried completely under mild sun and then ground into coarse powder with an electric grinder. The powdered material was then extracted with $96 \%$ ethanol at room temperature for 7 days with occasional shaking and stirring. The extract was filtered through cotton filter and the filtrate was then concentrated with a rotary evaporator under reduced pressure to get brownish mass that was kept in refrigerator for further use.

\subsection{Animal studies}

All protocols for the animal experiments were reviewed and approved by the animal care and use committee of Institute of Biological Science, University of Rajshahi. Long-Evans male rats weighing about 110-130 gm, aged 2 months, were purchased from animal's house of International Centre for Diarrhoeal Disease Research, Bangladesh (ICDDRB). Prior to commencement of the experiments, all the rats were acclimatized to the new environmental condition for a period of one week. During the experimental period, the rats were kept in a well ventilated animal house at room temperature and were supplied standard pellets from ICDDRB and fresh drinking water. All the rats were kept in cages and maintained with natural 12 hour light and dark cycle. Adrenaline was injected intraperitoneally (i.p.) in rats. Adrenaline-induced dyslipidemic rats (AIDRs) were treated with i.p. injection of ethanolic extract of $N S$ seed $(0.5,1$ and $1.5 \mathrm{mg} / \mathrm{kg} \mathrm{BW})$ for 2 weeks as dose adjustment. After completing the dose adjustment, AIDRs were treated with i.p injection of NSSE (1.5 mg/kg BW) and atorvastatin $(20 \mathrm{mg} / 70 \mathrm{~kg} \mathrm{BW})$ for 8 weeks.

\subsection{Measurement of lipid profile}

After completing the treatment with ethanolic extract of $N S$ seed and atorvastatin, the rats were at first anesthetized with sodium phenobarbital. Then abdominal skin was cut, and thoracic artery was opened. Finally, 3-5 ml of blood was collected directly from thoracic artery by syringes. At last, the blood was centrifuged at $4000 \mathrm{rpm}$ for 10 minutes, and the serum was obtained. Serum lipid profile, such as TC, TG, LDL-C and HDL-C, was assessed using diagnostic kits (Human, Germany).

\subsection{Free radical scavenging activity}

Free radical scavenging activity was determined using the modified method described by Blois [19]. The assay was performed by adding $20 \mu \mathrm{l}$ of blood plasma and $10 \mathrm{mM}$ sodium 
phosphate buffer (pH 7.4) to $400 \mu \mathrm{l}$ of $0.1 \mathrm{mM}$ methanolic solution of 1,1-diphenyl-2picrylhydrazyl (DPPH). After $30 \mathrm{~min}$ incubation at ambient temperature $\left(21^{\circ} \mathrm{C}\right)$, absorbance of the samples was measured at $517 \mathrm{~nm}$. As precipitate was formed in most of the plasma samples, they were centrifuged before the measurement.

\subsection{Histopathological analysis}

Histopathological studies were performed for the determination of LV weight and cardiomyocyte cross sectional area as reported previously [20-21]. In brief, LV weight was measured, and the ratio of LV weight to body weight was calculated to determine an index of LV hypertrophy. Four sections were obtained from each heart, and mounted on slides and stained with hematoxylin and eosin. To evaluate the extent of cardiomyocyte hypertrophy, cross sectional images of cardiomyocyte were scanned at $\times 400$ magnifications. Approximately 20 cross-sections of cardiomyocyte were analyzed in each heart. Average values for each heart were used for analysis. All images were taken using A. KRUSS OPTRONIC (Hamburg, Germany), and all measurements were determined using Scion Image software (Scion Corporation, Frederick, MD).

\subsection{Statistical analysis}

The results were expressed as mean \pm SEM (standard error of the mean). We used a oneway analysis of variance (ANOVA), followed by Dunnett's post-hoc test or students paired or unpaired $t$-test where appropriate. The statistical method applied in each analysis was described in each figure. Results were considered to be significant when $\mathrm{p}$ values were less than $0.05(\mathrm{p}<0.05)$.

\section{Results}

\subsection{Effects of Nigella sativa seed extract (NSSE) on lipid profile in AIDRs}

AIDRs were treated with ethanolic extract of $N S$ seed at different concentration $(0.5,1$ and $1.5 \mathrm{mg} / \mathrm{kg} \mathrm{BW}$ ) for two weeks to determine the appropriate dose at which most significant results were obtained and the determined dose was used to carry out the research for next 8 weeks protocol.

Induction of adrenaline in rats for two weeks significantly altered lipid profile when compared with normal rats (Fig. 1A-1D). We studied the effects of different doses of NSSE $(0.5,1$ and $1.5 \mathrm{mg} / \mathrm{kg} \mathrm{BW}$ ) on lipid profile (e.g. TC, TG, LDL-C and HDL-C levels) to select the most effective dose of NSSE in AIDRs. We observed that NSSE at different doses significantly changed the lipid profile and most significant change of lipid profile was found at $1.5 \mathrm{mg} / \mathrm{kg}$ BW among three doses (Fig. 1A-1D). The NSSE (1.5 $\mathrm{mg} / \mathrm{kg} \mathrm{BW}$ ) reduced TC level $1.25 \mathrm{mmol} / \mathrm{L}$ (Fig. 1A), TG level $1.19 \mathrm{mmol} / \mathrm{L}$ (Fig. 1B), and LDL-C level $1.46 \mathrm{mmol} / \mathrm{L}$ (Fig. 1C), whereas the NSSE increased HDL-C level 0.73 
mmol/L (Fig. 1D) when compared with AIDRs. As the effect of NSSE on lipid profile was dose dependent, we selected the most effective dose for further study.
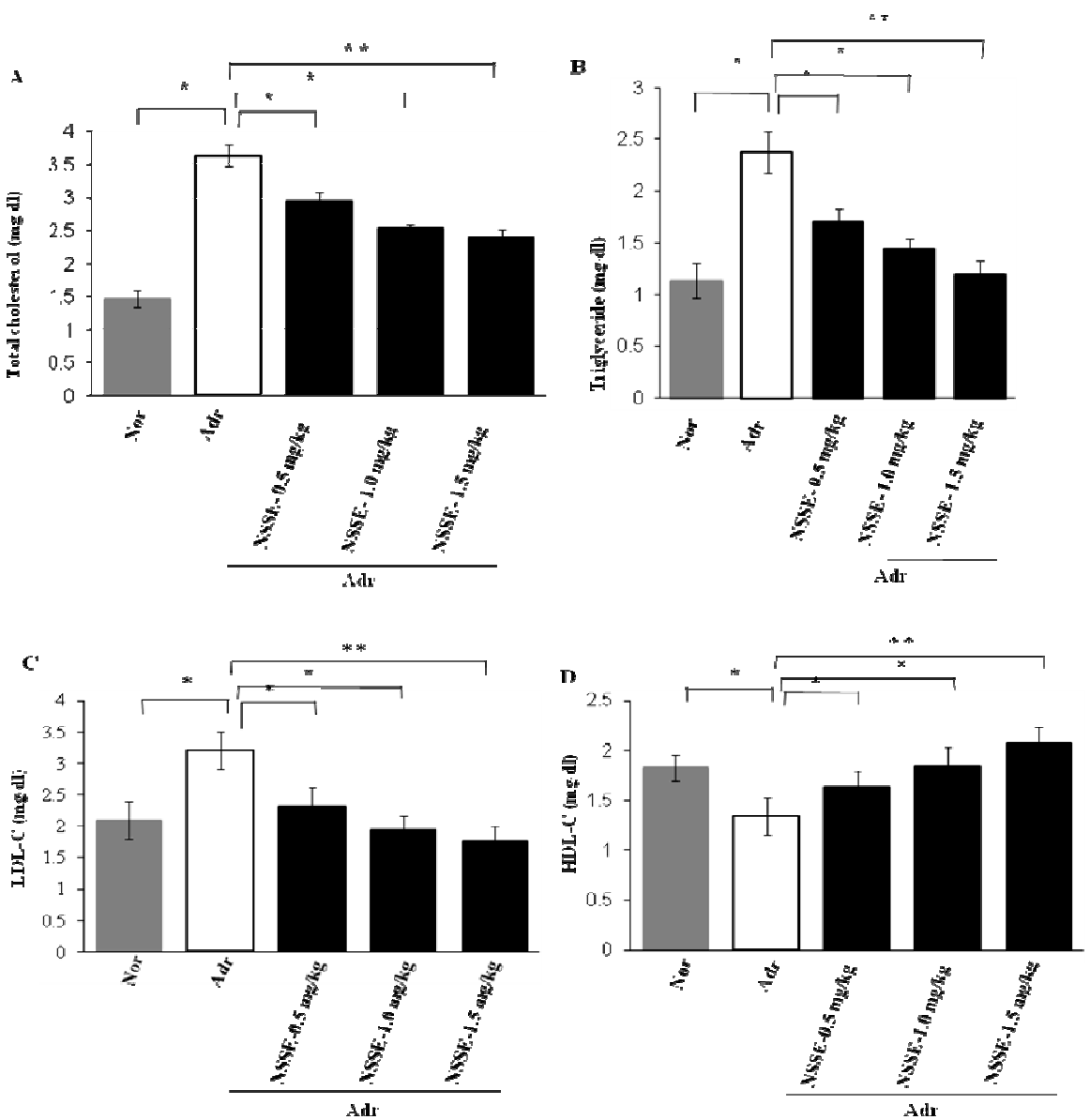

Fig. 1. Effects of NSSE of different doses on lipid profile [A) Total cholesterol, B) Triglyceride, C) Low-density lipoprotein-cholesterol and D) High-density lipoprotein-cholesterol] in AIDRs for two weeks. All values were presented as mean \pm SEM; $n=6$ in each group, ${ }^{*} p<0.05$ and $* * P<0.01$ compared to adrenaline-induced dyslipidemic rats (ANOVA followed by Dunnett's test). Nor = Normal, $\mathrm{Adr}=$ Adrenaline, NSSE $=$ Nigella sativa seed extract.

Like two weeks, induction of dyslipidemia by adrenaline in rats for 8 weeks significantly altered lipid profile when compared with normal rats (Fig. 2A-2D). To evaluate the effect of NSSE on lipid profile, we examined TC, TG, LDL-C and HDL-C after i.p injection of NSSE and atorvastatin (as reference standard) for 8 weeks in AIDRs. After eight weeks treatment with NSSE and atorvastatin, we found that both NSSE and atorvastatin reduced TC level $1.83 \mathrm{mmol} / \mathrm{L}$ and $1.98 \mathrm{mmol} / \mathrm{L}$ (Fig. 2A), TG level 1.02 
$\mathrm{mmol} / \mathrm{L}$ and $1.16 \mathrm{mmol} / \mathrm{L}$ (Fig. 2B), and LDL-C level $1.01 \mathrm{mmol} / \mathrm{L}$ and $1.04 \mathrm{mmol} / \mathrm{L}$ (Fig. 2C), whereas they increased HDL-C level $0.4 \mathrm{mmol} / \mathrm{L}$ and $0.5 \mathrm{mmol} / \mathrm{L}$ (Fig. 2D), respectively when compared with AIDRs. These results suggested that the effect of NSSE on lipid profile closely resembled to that of atorvastatin.
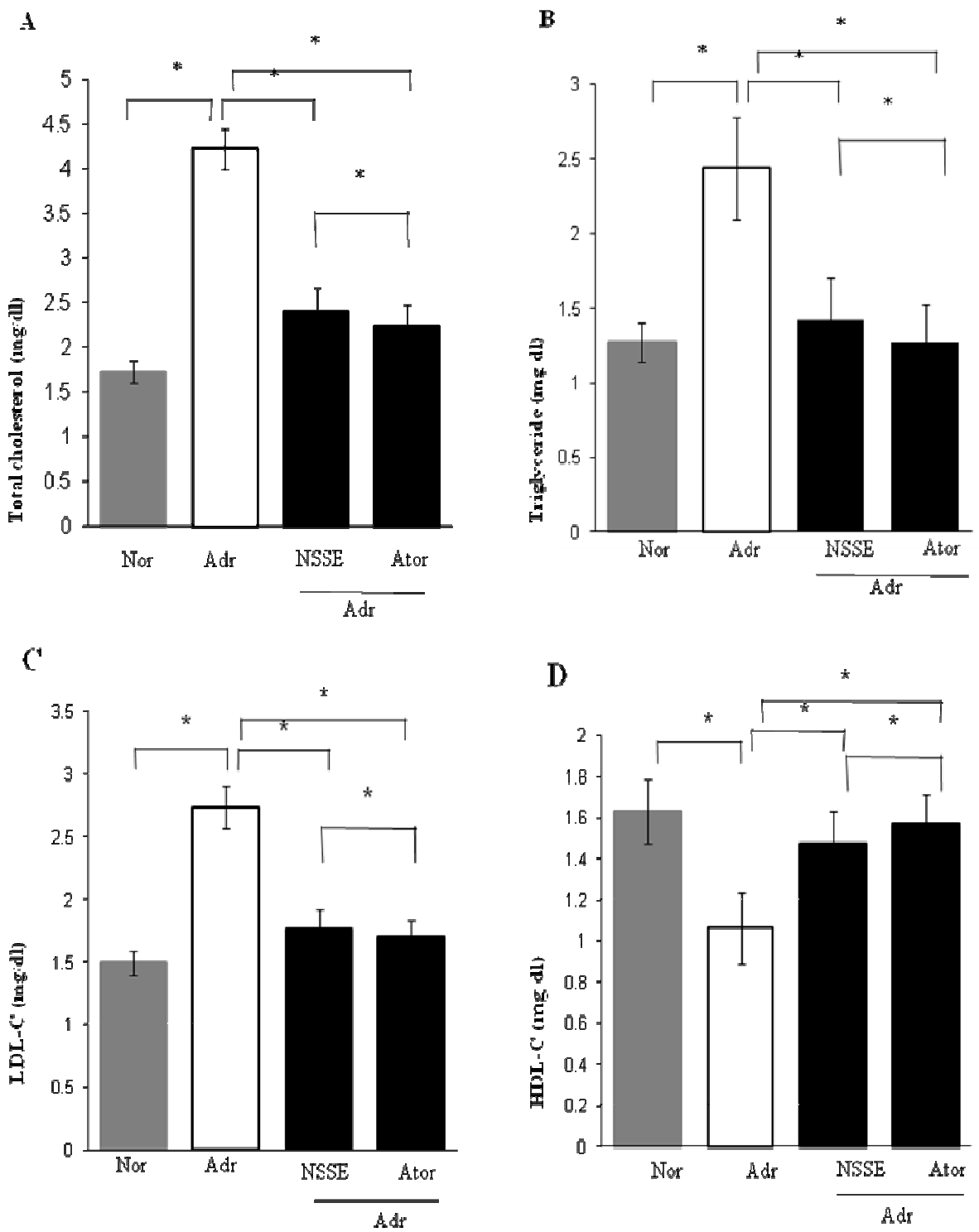

Fig. 2. Effects of NSSE and atorvastatin on lipid profile [A) Total cholesterol, B) Triglyceride, C) Low-density lipoprotein-cholesterol and D) High-density lipoprotein-cholesterol] in AIDRs for eight weeks. Data were presented as mean \pm SEM; $n=6$ in each group, ${ }^{*} p<0.05$ compared to eight weeks adrenaline-induced dyslipidemic rats (ANOVA followed by Dunnett's test). 


\subsection{Effect of NSSE on scavenging of DPPH free radicals in AIDRs for eight weeks}

To determine the effect of NSSE and atorvastatin on DPPH free radical, NSSE and atorvastatin were injected i.p. to AIDRs for eight weeks. The result showed that the percentage of free radical scavenging activity of NSSE and atorvastatin was 27.10 and 23.20 , respectively, which was increased by $80.31 \%$ and $53.97 \%$ respectively when compared with normal rats (Fig. 3A). This result revealed that NSSE had higher antioxidant activity than that of atorvastatin.

$\rightarrow$

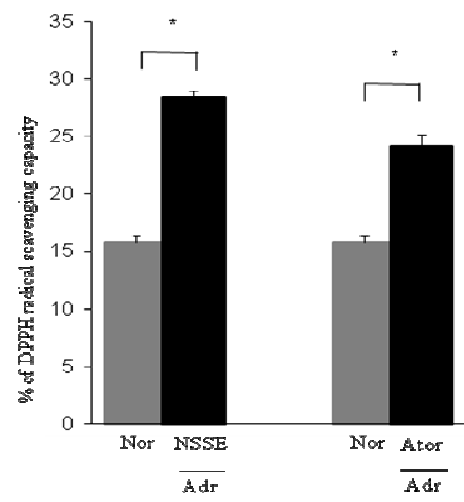

$\mathbf{B}$

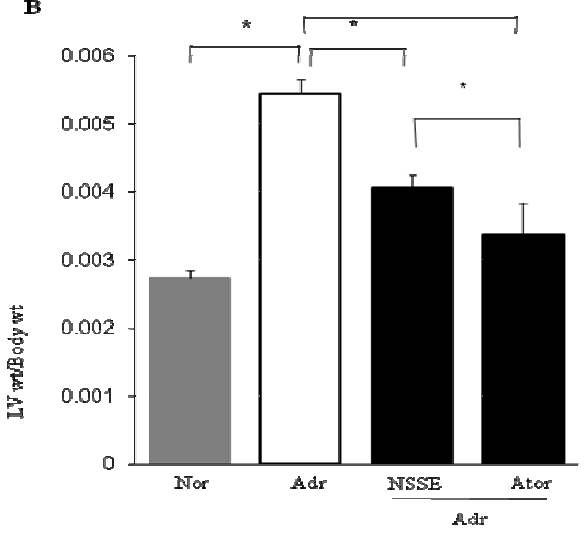

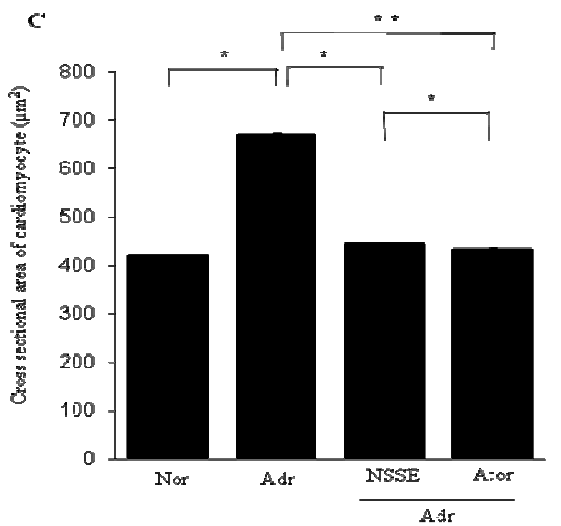

Fig. 3. Effects of NSSE and atorvastatin on A) DPPH radical scavenging activity, B) Left ventricular hypertrophy and C) Cardiomyocyte size in AIDRs. The data were shown as mean \pm SEM, $n=6$ in each case. $* P<0.05$ and $* * P<0.01$ compared to eight weeks adrenaline-induced dyslipidemic rats (ANOVA foll-owed by Dunnett's test).

\subsection{Effects of NSSE on LVH in AIDRs for eight weeks}

Intraperitoneal administration of adrenaline in rats for eight weeks significantly increased LVH (0.0055) compared with normal rats (0.0027). To know the inhibitory effect of 
NSSE and atorvastatin on LVH (LV weight/body weight), we treated the AIDRs with NSSE and atorvastatin for 8 weeks. After 8 weeks treatment, NSSE significantly reduced LVH (0.0040) when compared with AIDRs. Atorvastatin also significantly reduced LVH (0.0034). This finding suggested that the NSSE effectively reduced LVH and the inhibitory effect of NSSE on LVH was similar to that of atorvastatin (Fig. 3B).

\subsection{Effects of NSSE on cardiac myocyte size in AIDRs for eight weeks}

Injection of adrenaline in rats for eight weeks significantly increased cardiac myocyte cross sectional area $\left(668.65 \mu \mathrm{m}^{2}\right)$ when compared with normal rats $\left(421.30 \mu \mathrm{m}^{2}\right)$. To find out the inhibitory effects of NSSE and atorvastatin on cardiac myocytes size, we treated the AIDRs with NSSE and atorvastatin for 8 weeks. NSSE significantly reduced cardiac myocyte size $\left(444.43 \mu \mathrm{m}^{2}\right)$ when compared with AIDRs. Atorvastatin also significantly reduced cardiac myocyte cross sectional area $\left(433.17 \mu \mathrm{m}^{2}\right)$. Our result inferred that NSSE and atorvastatin equally inhibited cardiac myocytes size (Fig. 3C).

\section{Discussion}

Atherosclerotic cardiovascular and cerebrovascular diseases are the major cause of disability in western as well as in developing countries [22]. Dyslipidemia following CVD is obviously a grave consequence. Prevalence of different forms of CVD with their associated complications such as cardiohypertrophy is increasing, but the treatment alternatives are limited due to unwanted side effects and high costs [23]. In the recent years, medicinal plants have come to view for the treatment of many diseases including CVD owing to their easy availability and relatively minimal adverse effects. Due to advancement of modern technology, isolation and characterization of bioactive compounds from natural sources could be made possible. Therefore, it could be expected that the successful trials may lead to isolation of more potent therapeutically effective drugs from medicinal plants having relatively less adverse effects. From our present investigation $N S$ is found to be effective medicinal plant in the treatment of dyslipidemia and LVH in rats.

$N S$ is available in the South-Asian region whose alleviating effects upon several ailments have been noticed from long time [24]. Saha et al (2004) [25] has been shown that NSSE possessed significant lipid lowering effects in rats suggesting that treatment with NSSE in sufficient amount might lower dyslipidemia. A significant increase in TC, TG and LDL-C levels and decrease in HDL-C level were observed in our experiment after induction of dyslipidemia by adrenaline for both two and eight weeks protocol. The NSSE significantly reduced the TC, TG and LDL-C levels, and increased the HDL-C level and the results were consistent with the previously published reports [26-27]. The hypolipidemic effect of NSSE was due to the synergistic action of its different constituents, including thymoquinone, soluble fiber, sterols, flavonoids and high content of polyunsaturated fatty acids [28]. Although the exact mechanism of hypolipidemic 
action is not clear, it had been hypothesized that NSSE produced anti-dyslipidemic effect by decreasing cholesterol synthesis, and more importantly by having antioxidant properties.

It has been reported that dyslipidemia increased oxidative stress and oxidative stress had been implicated in the pathogenesis of a variety of human diseases including CVD [29]. Since NSSE had hypolipidemic effect, therefore NSSE might be used in the treatment of CVD caused by oxidative stress. We observed in our study that NSSE significantly reduced oxidative stress by scavenging the free radicals (Fig. 3A). Thus, we conclude that NSSE had appreciable free radical scavenging properties and our data are agreed with previous studies [30].

Chronic dyslipidemia is associated with CVD. It has been reported that i.p injection of adrenaline in rats for several weeks produced LVH [31]. However, recent evident revealed that LVH was associated with enhanced oxidative stress by up-regulation of endothelial $\mathrm{NAD}(\mathrm{P}) \mathrm{H}$ oxidase and endothelial dysfunction [32]. In our present study, i.p. injection of adrenaline for 8 weeks markedly increased the left ventricle weight/body weight ratio and NSSE significantly reduced LVH (Fig. 3B). The inhibitory effect of NSSE on LVH may due to the fact that the reduction of oxidative stress was done by NSSE.

Statin had pliotropic effect for reducing cardiovascular hypertrophy $(\mathrm{CVH})$ in vivo through inhibition of Rac1 signaling pathway [32]. The present study demonstrated that induction of dyslipidemia by adrenaline increased the cardiomyocyte size in rats. Both standard drug and NSSE were effectively ameliorated adrenaline-induced increased cardiomyocyte size in vivo (Fig. 3C). The inhibitory effect of NSSE on cardiomyocyte size suggested the therapeutic potential of NSSE for the treatment of CVH.

In conclusion, the present study suggested the ethanolic extract of $N S$ seed as a potent lipid lowering and antioxidant agent. Also, the activity to reduce the LVH may contribute to its cardio protective role. Further studies are necessary to insight the inhibitory effect of NSSE in molecular level on LVH.

\section{Acknowledgement}

The authors thank Square Pharmaceuticals Ltd, Bangladesh for its kind gift of atorvastatin and metformin. The authors also thank Professor Dr. Abdur Rashid, Faculty of Pharmacy, Department of Pharmaceutical Chemistry, University of Dhaka, for his generous gift of DPPH.

\section{References}

1. A. R. Jaffar, J. Babb, and A. Movahed, Int. J. Cardiol. 97, 355 (2004). http://dx.doi.org/10.1016/j.ijcard.2003.07.039

2. H. J. Yoon, M. H. Jeong, J. H. Bae, K. H. Kim, Y. Ahn, J. G. Cho, J. C. Park, and J. C. Kang, Korean Circ. J. 41, 124 (2011).

3. W. B. Kannel and P. W. F. Wilson, Cardiovascular risk factors and hypertension, 3rd edn. (American Heart Association, Dallas, 2003) pp. 35-238.

4. K. Prasad, Atherosclerosis 179, 269 (2005). http://dx.doi.org/10.1016/j.atherosclerosis.2004.11.012 
5. R. Scragg, R. Jackson, I. Holdaway, G. Woollard, and D. Woollard, Am J Cardiol. 64, 971 (1989). http://dx.doi.org/10.1016/0002-9149(89)90792-3

6. N. Ara, M. Rashid, and M. S. Amran, Pak. J. Pharm. Sci. 22, 267 (2009).

7. E. I. Boesen, W. P. Andersion, M. K. Michelle, and J. Hyper, 23, 987 (2005).

8. N. Naher, A. H. M. K. Alam, S. Islam, Al-Mamun, A. K. M. M. Hossain, M. A. A. Rahman, and M. Rashid, Bang. Pharm. J. 15, 135 (2012).

9. S. J. Thandapilly, X. L. Louis, T. Yang, D. M. Stringer, L. Yu, S. Zhang, J. Wigle, E. Kardami, P. Zahradka, C. Taylor, H. D. Anderson, and T. Netticadan, Eur. J. Pharmacol. 668, 217 (2011). http://dx.doi.org/10.1016/j.ejphar.2011.06.042

10. B. J. Kallfelt, A. P. Waldenstrom, C. Hjalmarson, J. Mol. Cell. Cardio. 9, 383 (1997). http://dx.doi.org/10.1016/S0022-2828(77)80005-9

11. M. J. Hoeg, R. E. Gregg, and B. H. Brewer, J. A. M. A. 255, 512 (1986). http://dx.doi.org/10.1001/jama.1986.03370040086030

12. M. S. Islam, A. H. M. K. Alam, M. A. A. Rahman, Y. Ali, A. Mamun, M. Rahman, A. K. M. M. Hossain, and M. Rashid, J. Sci. Res. 4, 709 (2012). doi: http://dx.doi.org/10.3329/jsr.v4i3.9974

13. P. H. Davis, Flora of Turkey and the East Aegean Islands (Edinburgh Univ. Press: Edinburgh, England, 1965) pp. 98-105.

14. M. L. Salem, Int. Immunopharmacol. 5, 1749 (2005). http://dx.doi.org/10.1016/j.intimp.2005.06.008

15. S. H. Swamy and B. K. Tan, J. Ethenopharmacol. 70, 1 (2000).

16. M. A-Al Atef and W. A. A. Taisan, Aust. J. Basic Appl. Sci. 4, 957 (2010).

17. A. Zahoor, A. Ghaffar, and M. Aslam, Agric. Livestock Pak. 6 (2004).

18. A. H. M. K. Alam, H. Rashid, M. A. A. Rahman, A. Baki, and G. Sadik, Pak. J. Biol. Sci. 5, 1264 (2002). http://dx.doi.org/10.3923/pjbs.2002.1264.1266

19. M. S. Blois, Nature, 181, 1199 (1958). http://dx.doi.org/10.1038/1811199a0

20. Y. Mukai, H. Shimokawa, T. Matoba, T. Kandabashi, S. Satoh, J. Hiroki, K. Kaibuchi, and A. Takeshita, FASEB J. 15, 1062 (2001).

21. H. Midoriko, H. Shimokawa, T. Hattori, J. Hiroki, Y. Mukai, K. Morikawa, T. Ichiki, S. Takahashi, and A. Takeshita, Circ Res. 93, 767 (2003). http://dx.doi.org/10.1161/01.RES.0000096650.91688.28

22. S. Aqil, A. Jaleel, F. Jaleel, and F. Basir, World Appl. Sci J. 3, 759 (2008).

23. R. DiBianco, Am. J. Med. 115, 480 (2003). http://dx.doi.org/10.1016/S0002-9343(03)00265-1

24. M. T. Al-Hariri, T. Yar, A. O. Bamosa, and M. N. El-Bahai, J. Pak. Med. Assoc. 59, 363 (2009).

25. R. R. Saha, Z. F. Dewan, and N. Uddin, Bang. J. Physol. Pharmacol. 20, 36 (2004).

26. M. El-Dakhakhny, N. I. Mady, and M. A. Halim, Arzneimittel Forsch. 50, 832 (2000).

27. N. Tasar, O. Sehirli, O. Yiginer, S. Suleymanoglu, M. Yuksel, B. Yegen, G. Sener, Marmara Pharm. J. 16, 141 (2012).

28. B. H. Ali and G. Blunden, Phytother. Res. 17, 299 (2003). http://dx.doi.org/10.1002/ptr.1309

29. B. Halliwell and J. Gutteridge, Free Radicals in Biology and Medicine, 2nd ed. (Oxford University Press, New York, 1989).

30. M. Burits and F. Bucar, Phytother. Res. 14, 323 (2000). http://dx.doi.org/10.1002/1099-1573(200008)14:5<323::AID-PTR621>3.0.CO;2-Q

31. H. Hus, Y. Le, and M. Chen, Prostag. Other Lipid Mediat. 66, 99 (2001).

32. M. Rashid, S. Tawara, Y. Fukumoto, M. Seto, K. Yano, and H. Shimokawa, Circulation J. 73, 361 (2009). http://dx.doi.org/10.1253/circj.CJ-08-0817 